\title{
SportsWoman: A semantic-based recommender platform for women who practice sports
}

\author{
Juan M. Santos-Gago*, Luis Álvarez-Sabucedo*, Víctor M. Alonso-Rorís ${ }^{\dagger}$, José L. García-Soidán ${ }^{\ddagger}$, Carmina \\ Wanden-Berghe ${ }^{\S}$, and Javier Sanz-Valero \\ ${ }^{*}$ School of Telecommunication Engineering. Campus Lagoas-Marcosende 36310 Vigo, Spain \\ ${ }^{\dagger}$ DataSpartan. Av. Otero Pedrayo 15570 Narón, Spain \\ ${ }_{\ddagger}^{\ddagger}$ Faculty of Education and Sports Sciences. Campus A Xunqueira 36005 Pontevedra, Spain \\ ${ }^{\S}$ Health and Biomedical Research Institute of Alicante, University General Hospital of Alicante, Pintor Baeza \\ 03010 Alicante, Spain \\ `Faculty of Medicine, Miguel Hernández University, Sant Joan D’Alacant 03550 Elche, Spain
}

\begin{abstract}
This article introduces SportWoman, a telematic application aimed at giving support in the form of recommendations to women who practice sports, whether professional or amateur or even sporadic practitioners of sports or fitness activities. The platform tries to cover the gap of woman personalization left by mobile applications supporting sports and fitness that currently exist in the market by taking into account the menstrual cycle of women and how this cycle affects them individually. SportsWoman was designed as an Expert System in which information is stored in a Knowledge Base using semantic technologies. In the proposed platform, the knowledge of specialists (physicians and researchers of sports science) is expressed using a particular ontology and a set of rules that, in turn, determine the daily recommendations for each user. SportsWoman has been tested and evaluated by 34 athletes through the well-known System Usability Scale, obtaining a value of 86, which corresponds to an acceptable level of usability with a grade $B$.
\end{abstract}

Index Terms-Sports, Women, Menstrual Cycle, Expert System, Semantic Technologies, Ontologies, Inference Rules.

\section{INTRODUCTION}

$\mathbf{I}$ $\mathrm{N}$ recent years, tools for monitoring sports activities have become popular. Both professional and amateur athletes, and even people who perform physical activities sporadically, often make use of mobile devices and applications that allow recording training sessions. This information is beneficial to know the sports performance of the user and serves as a support to set or adapt the training routines and, in this way, to achieve the pursuit sport or fitness goals.

Many of these tools are sophisticated. They allow collecting information of different nature that can be used to customise training plans according to the particular conditions of the athletes at each moment. In most cases, these tools consist of applications that run on mobile devices (smartphones and smartbands) and collect data from sensors within these devices, such as location, altitude, cadence, but also heart rate when using pectoral bands or appropriate smartbands. Also, the athletes themselves introduce data manually (such as weight, height, perceived effort after performing a physical activity, mood, injuries produced, among others). All this information characterise the actual state of the athlete and determines what the training should be like in each moment. It is clear that the physical condition and the mental status (such as stress or depression) drives sports performance. So these conditions are taken into account, especially those that refer to the physical state, by the current sports monitoring tools. However, to this day, there are no tools in this category that make use of information about the menstrual cycle to make personalised recommendations specific to women.

Different studies have highlighted the interrelation between the menstrual cycle and sport. On the one hand, high-intensity training can cause disorders in the menstrual cycle [1], [2]. On the other hand, the cycle can influence the performance of athletes [3], [4]. The hormonal change linked to the menstrual cycle involves significative changes in the body and the mood of women. Although the effects of these changes manifest themselves very differently from one woman to another, none is free from them. And although in most cases the influence of these changes does not dramatically affect the performance in sports, it is advisable to adjust the type and intensity of training depending on the menstrual cycle and how this cycle particularly affects each particular athlete. Generically, during the follicular phase, i.e., the first half of the cycle, there is a progressive increase in estrogen, it increases pain tolerance, resistance, and the ability to exert force. In turn, the luteal phase, i.e., the second half of the cycle, is characterised by a progressive increase of progesterone and a decrease in estrogen and serotonin. Also, it can be noted an increase in body temperature and fluid retention. In the luteal phase, hormonal changes can lead to fatigue and less ability to exert force. Therefore, it is typically advisable to introduce higher training loads towards the second and third week of the menstrual cycle, and reduce them during the week before the period. Although this will depend to a large extent on how the cycle affects each woman in particular.

This article presents SportsWoman, a telematics platform whose objective is to facilitate the monitoring and collection of information related to the menstrual cycle and, based on it, generate daily personalised recommendations for women who practice sports. The article is an extension of the work [5], which was previously presented at the 7th World Conference 
on Information Systems and Technologies (WorldCist'19). The article is organised into 7 sections. Section II includes a brief description of the study of the state of the art that was carried out in order to identify solutions related to the ideas above mentioned. Sections III and IV present the conceived proposal and its architecture. Section V describes the ontology that supports the description of facts in the knowledge base of the platform. Section VI explains the validation process that has been carried out. Finally, section VII briefly discusses the results obtained and the future lines of the work.

\section{StATE OF THE ART}

The achievement of the objective raised in this research departs from an initial study of the state of the art related to technological solutions aimed at training support for female athletes that take into account their menstrual cycle. In this sense, a systematic review was carried out, on the one hand, of the commercial tools available in mobile app stores such as Google Play or App Store and, on the other hand, of works published in the scientific literature.

Concerning commercial software solutions, it is worth mentioning the existence in the market of a multitude of applications aimed at monitoring and analysing sports activities. Remarkable companies of sports equipment and manufacturers of smartphones, smartbands or smartwatches have made available to amateur and professional athletes products specifically aimed at the management of sports activities and fitness. These products range from simple applications that allow registering sports activity, to sophisticated solutions to analyse these activities in detail and even provide recommendations focused on improving performance. However, the number of tools that take into account the particularities of women is minimal and, among them, those considering the menstrual cycle are practically anecdotal.

Among the applications intended to be used by women, taking into account its popularity and the assessment provided by its users, the following three are worth noting: Female Fitness - Women Workout [6], Workout for Women - Weight Loss Fitness App [7] and Women Workout: Home Fitness, Exercise \& Burn Fat [8]. These apps are downloaded by millions of women and are valued very positively in stores. In essence, they are tools that suggest exercise routines aimed at reducing weight and working on certain parts of the body that may be of greater interest to women. In some cases this kind of apps are useful tools to record training and motivate users. However, they are simple and have little capacity for personalisation and adaptation to the peculiarities of a particular female athlete. And more importantly, none of them takes into account the menstrual cycle in the proposal of exercise routines.

A more remarkable tool with greater potential in this sense is the platform for sports activities management from Fitbit [9]. In mid-2018, Fitbit announced the launching of a new service for its app aimed at supporting women health. This service facilitates the registration of generic parameters about the menstrual cycle and its symptoms. Fitbit specifies that it is oriented "to help you learn more about your menstrual cycle -and your body- so you can better understand how it affects other aspects of your health and fitness" [10]. However, to this day, the information collected about the menstrual cycle is not used to adapt the functions offered by its platform.

The new service offered by Fitbit was studied in the scope of our research project in order to identify useful elements of characterisation of the menstrual cycle. Other popular menstrual cycle management applications in mobile stores, such as Period Tracker, Ovulation Calendar \& Fertility [11], Period Tracker Flo, Pregnancy \& Ovulation Calendar [12] o Period Tracker: Monthly Cycles [13], were also analysed with that same purpose. Although the latter are not specifically intended for the sports domain, they help women to trace their menstrual cycles and facilitate a successful conception (or, where appropriate, pregnancy avoidance). The apps allow recording daily parameters such as colour or flow of the menstruation, temperature of the woman, related symptoms or mood.

With regard to the analysis of works related to our proposal in the scientific literature, the search in the most relevant databases was unsuccessful. This search was carried out in the following repositories: Google Scholar, Springer Link, MEDLINE through PubMed and JMIR Publications. The query launched on the different search engines was: (menstruation OR menstrual) AND (sport OR fitness) AND (software OR application OR app). As a result, no publication describing any proposal similar or related to ours or that studied any analogous system was obtained. Hardly, a very limited number of papers dealing with the subject tangentially could be identified. Among the latter is the work of Sohda et al. [14], which presents a study aimed at clarifying how the data obtained from a self-tracking health app for female users (an app similar to the aforementioned for the management of the menstrual cycle) can be used to improve the accuracy of prediction of the date of next ovulation.

From the study of the state of the art conducted, we can conclude the nonexistence of a platform like the one postulated in the previous section. While there are systems that recommend exercise routines specifically for women who practice sports (mainly casual sportswomen), these are very simple tools that do not take into account the current phase of the menstrual cycle or how this cycle specifically affects them.

\section{SPORTSWOMAN PlatForm}

The SportsWoman telematics platform was conceived within the scope of a multidisciplinary research project, involving researchers in the fields of sports science, medicine and telematics. The final aim is to support woman's specific sports practice, mainly professionals, but also amateurs or sporadic practitioners. The platform has a double purpose: i) it is aimed to facilitate the collection of data that allow characterising the menstrual cycle of a woman and determine how this cycle influences the physical and emotional state of the woman, as well as her sports performance; and ii) it is also designed to provide personalised recommendations to women regarding their sports training, taking into account, among other aspects, the particularities of their menstrual cycle. 


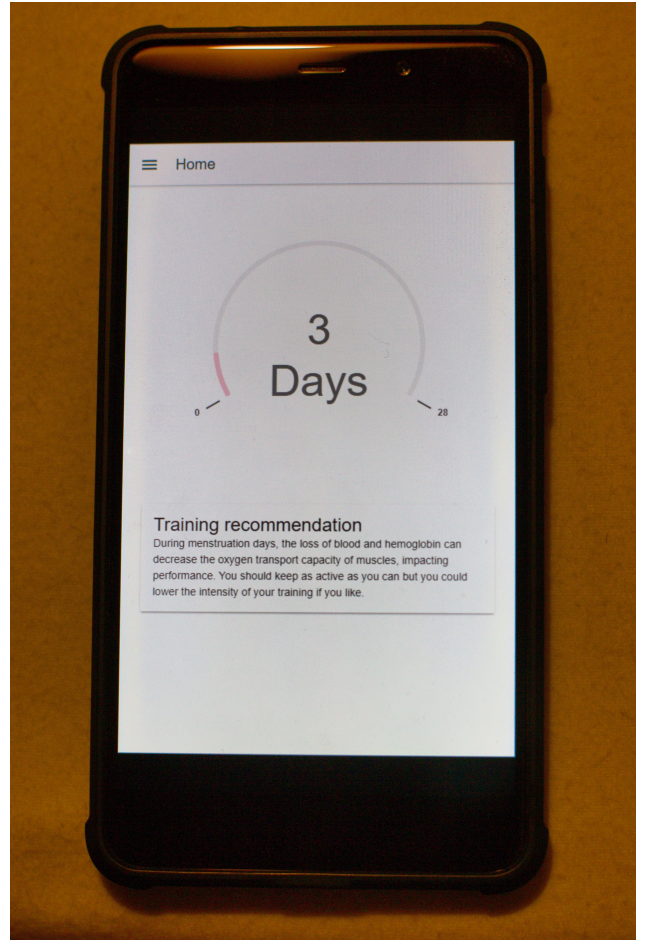

Fig. 1. SportsWoman user interface. Initial screen.

For the user interface (UI) of the platform, it was opted for a sober and functional design, in which the necessary options and functionalities are shown with the least possible number of distractions. To make use of the platform, a woman must register using a web form intended to collect data of a static nature, such as demographic data, physiological parameters, history of the menstrual cycle and habitual symptomatology, parameters related to rest and general characteristics of the sports activity practised. It should be noted that the data is stored anonymously on the platform. Once registered, the user accesses the platform through a Progressive Web App [15], typically through a mobile device, but could also do so through the web. On the initial screen of the app (cf. Fig. 1), it is presented the actual point in her menstrual cycle (either the real one, if the necessary data is registered, or the estimated point according to the previous history), as well as an estimation about the starting of the following cycle. Also, a list with the personalised recommendations deduced by the platform for that day is shown.

To add new data, see previously recorded data, or modify it, the user accesses a calendar (cf. Fig. 2). The calendar allows visualising with colour codes the evolution of previous menstrual cycles, as well as an estimate of future cycles. In addition to this information, it is also shown if there is data recorded for each day. The user can select the day for which she wants to enter data or modify already existing one (cf. Fig. 3). Through these services, users can register five categories of data: i) data related to menstruation, ii) biometric and physiological data, iii) information regarding the training exercises carried out, iv) data of medical tests, both blood and urine and, v) physiological and psychological

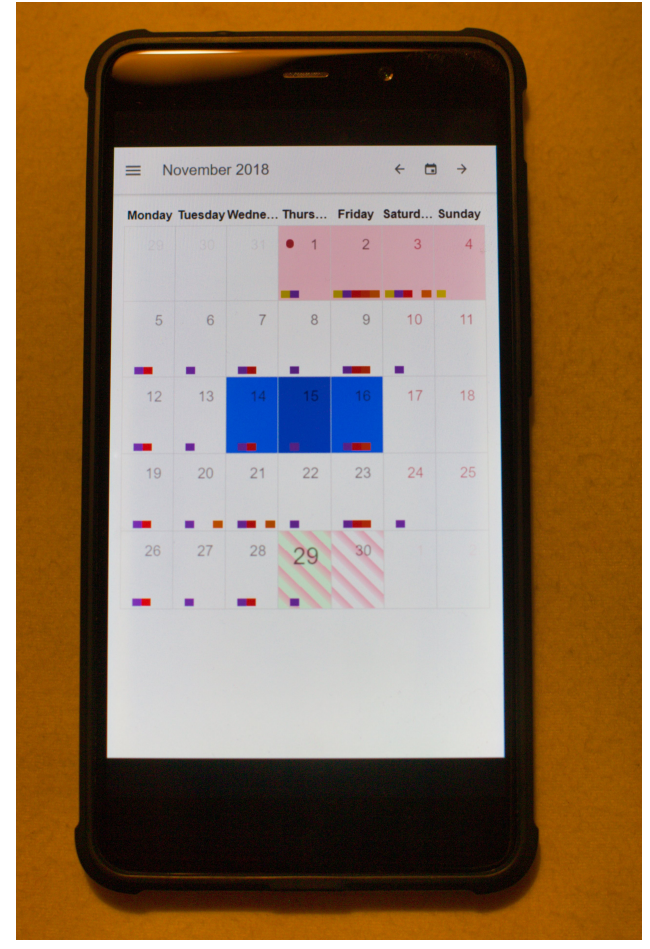

Fig. 2. SportsWoman User Interface. Calendar.

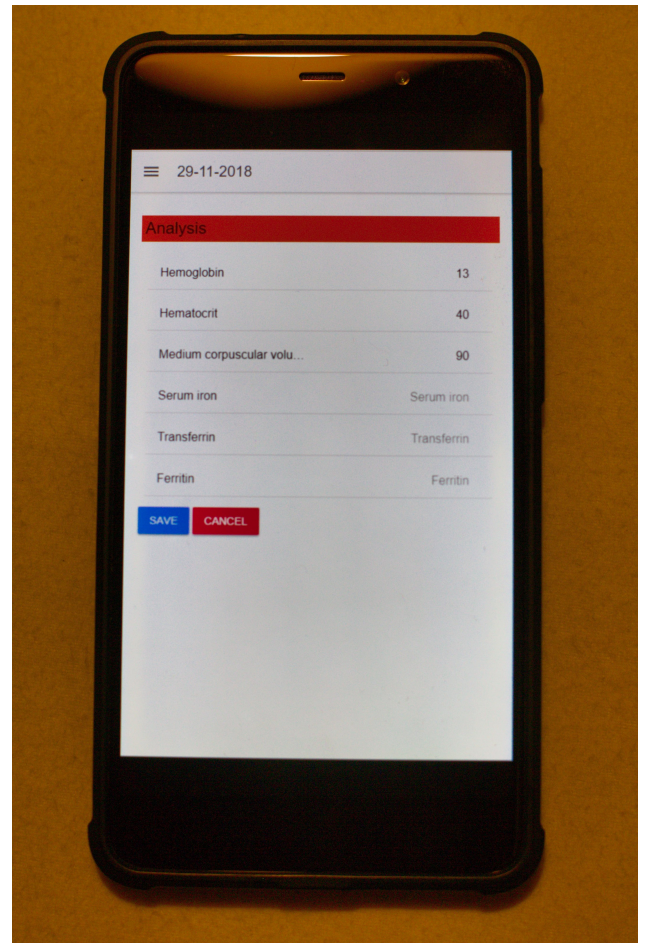

Fig. 3. SportsWoman User Interface. Medical variables form.

symptomatology.

All this information is used to accurately characterise each woman's menstrual cycle and estimate how this cycle affects her physical and psychological condition. The app also offers functionality for the visual representation of the evolution of different parameters, such as weight, body mass index or 
hematocrit, among several others. This functionality is very appreciated as it presents simply and graphically the trends of these parameters.

\section{Sports Woman Architecture}

The SportsWoman platform has been devised as a Knowledge-Based System (KBS) [16], i.e. a software solution that includes an explicit knowledge base (KB) containing a collection of information in a given field. And more specifically, it was designed as an Expert System [17], containing the $\mathrm{KB}$ the accumulated human expertise in the field and a set of if-then rules that can be used by an inference engine to make decisions or provide advises. Accordingly, information is mainly stored using semantic technologies, and part of the knowledge is stored as logic rules defined by specialists in the domain. These rules determine the recommendations presented to the user.

The authors have successfully applied this design model in several systems for different fields [18], [19], [20]. Based on these previous experiences, it was considered an appropriated approach as it allows to include new rules and new conceptual elements in the platform without making significative changes in the code. Following the construction paradigm of KBSs, the main architectural components of the platform are the following (cf. Fig. 4):

- Knowledge Base (KB). This component records all the information required for the proper functioning of the platform. This includes both factual information, i.e. the data registered daily by the users, as well as nonfactual information, that is registered using a Semantic Model. Nonfactual information forms a Semantic Model composed of two elements:

- Ontology. It represents the conceptual knowledge of the domain in the form of RDF/OWL triples. This element manages the terms used to describe and represent the domain knowledge, including computerusable definitions of basic concepts and the relationship among them. In section $\mathrm{V}$ the construction process of this artifact is discussed as well as its main characteristics.

- Rule sets. This element represents the heuristic information of the domain. It is made up of two sets of rules. The main group includes the rules about the recommendations daily presented to the users. This set of rules has been defined and agreed upon by a group of domain specialists, in particular, by experts in Medicine and Sports Science. It is a dynamic set of rules, which grows as new information is discovered. A second set of rules determines the users' monitorable parameters.

- Recommendations Manager. It is the component responsible for generating personalised recommendations for users. A logic rule execution engine guides its behaviour. This engine applies to the set of factual data in the knowledge base the first rule set above mentioned. This process generates a potential list of recommendations that are appropriate at that moment. The recommendations are prioritised; and only the first one, or a reduced number of them, is presented to the user. As an example, Table I shows some rules, expressed in logical notation (in the KBS are stored following the Node.JS JSON-rules-engine model). These rules would trigger the generation of the $\mathrm{R} 12$ recommendation when the user is in the last third of the luteal phase, and she is at risk of anemia, either due to having a low hemoglobin level or having had high bleeding in the last menstruation. Subsets of similar rules, defined for different points of the menstrual cycle, lead to the generation of other recommendations.

- Client Communication Interface. REST API to access the features within the KBS from a Client Agent. This API follows the scheme of verbs and HTTP addressing described in Table II.

- Personalisation Manager. One of the design premises of the proposed platform is to ensure that the interaction interfaces in the client are dynamic. This means that the parameters included in the data forms (cf. Fig 3) can be changed and adapted as the needs of the system evolve. A basic example of personalisation occurs according to the phase of the menstrual cycle in which the user is located: when the user declares to start the menstruation, the manager includes among the parameters to be monitored those related to bleeding (e.g., viscosity, colour, end of menstruation, among others). The mechanism of operation of this component is similar to that of the Recommendation Manager.

- Authorisation Server. An OAuth [21] server authenticates the communication between the Client Agent and the KBS. This server is usually a trusted third party, such as Google. In this way, the KBS can anonymise the recorded data, just by linking it with a non-personal code provided by the OAuth server. Meanwhile, the Client Agent can customise the user session with the user profile data in the authorisation service, such as the name or the photo, among others.

- Client Agent. This component was developed as a Progressive Web Application. In this way it is easy to provide support for a variety of popular smartphones both in iOS or Android systems. The main functional elements of the Client Agent are:

- Views Manager. Functional element responsible for generating the user interfaces at runtime. The parameters required for the monitoring forms are retrieved from the KBS (see the Personalisation Manager). To generate a form it receives a JSON object in which each monitorable parameter is described (name, datatype, description, etc.) according to the current conditions. This element processes this description, so that if there is any parameter that can be autocompleted, it does it automatically (e.g., current location). Using the remaining parameters, a graphical interface for the final user is generated.

- Persistence Manager. Element responsible for improving the performance, avoiding, if possible, invoking services from the KBS and guaranteeing the 


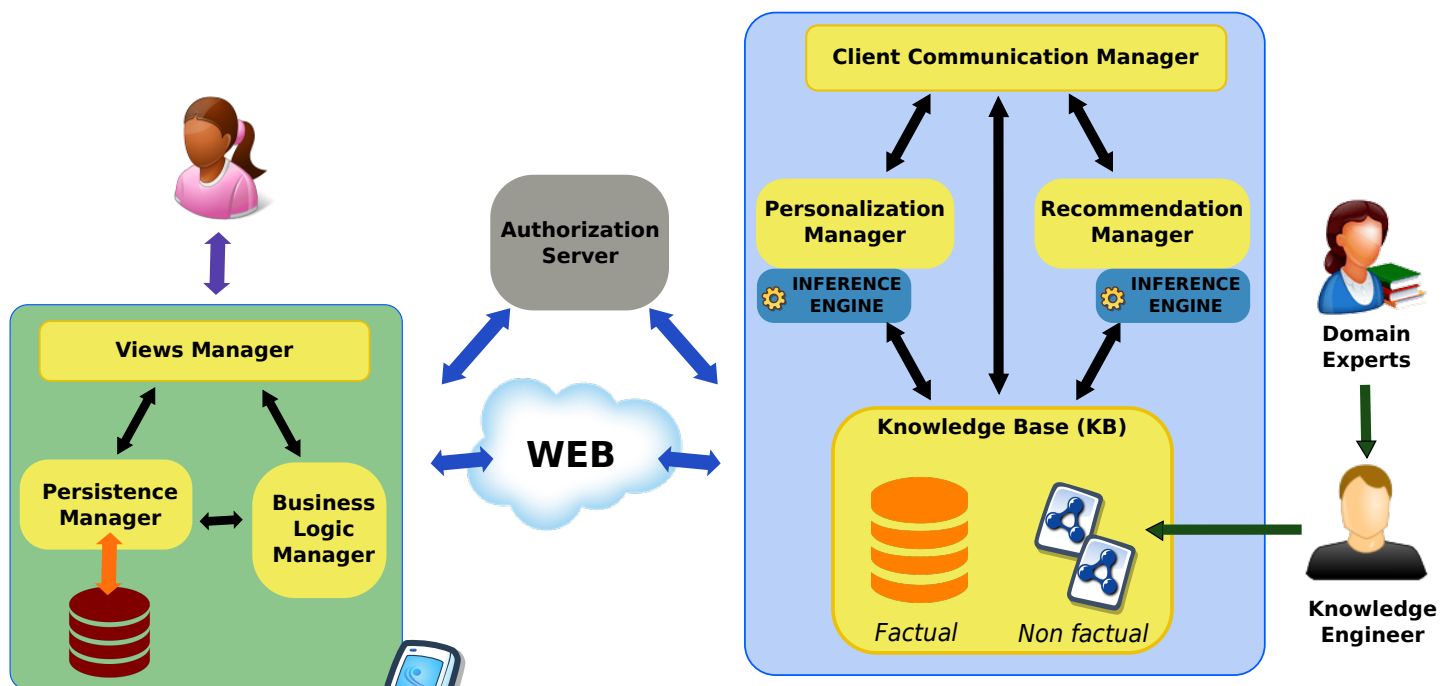

Client Agent

Kwoledge-Based System (KBS)

Fig. 4. SportsWoman architecture.

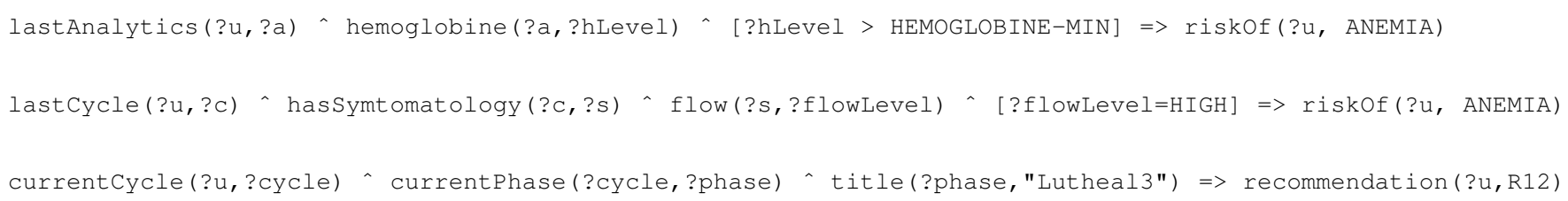

TABLE 1

EXAMPLE OF LOGICAL RULES.

TABLE II

KBS CLIENT ACCESS REST API

\begin{tabular}{lll}
\hline HTTP & URI & Description \\
\hline GET & /params & Get the list of monitorizable params \\
GET & /param/\{key $\}$ & Get information about a param \\
GET & /users $/\{$ id $\}$ & Get user info \\
GET & /users $/\{$ id $\} /$ logged_value & Get user param values (by dates $)$ \\
POST & $/$ users $/\{$ id $\} /$ logged_value & Send params for a specific user-date \\
DELETE & /users/id/logged_value & Remove param values by user-dates \\
GET & /users $/\{$ id $\} /$ logged_value $/\{$ key $\}$ & Get the user historic information about one \\
& & param \\
DELETE & $/$ users $/\{$ id $\} /$ logged_value $/\{$ key $\}$ & Remove all the param values \\
GET & /users $/\{$ id $\} /$ recommendations & Get the list of recommendations \\
\hline
\end{tabular}

operativeness of the client agent in offline mode.

\section{SPORTSWOMAN ONTOLOGY}

As mentioned in the previous section, the SportsWoman Knowledge Base is populated, on the one hand, with rules defined by domain experts, and on the other hand, with facts provided by the users. These facts are expressed in RDF/OWL using the terminology defined within the ontology developed ad-hoc for this platform.

An ontology identifies the concepts and relationships among them that exist in a given Universe of Discourse and constitutes a vocabulary that allows expressing facts in that universe. Building a semantic model and, in particular, an ontological model is a complex task, and different methodological approaches are possible. In this case, the authors followed a methodology already applied previously in several projects (such as [22], [23]), which yielded satisfactory results. This methodology is strongly based on Methontology/NEON [24], [25]. Nevertheless, in order to adapt it to our specific needs and taking into account previous experiences from the authors in software application development, it was decided to simplify and reshape some aspects, taking into account features from other methodologies such as DILIGENT [26], and UPON [27].

The construction process begins with the Specification Phase. The objectives of this phase are: to clarify the use that will be given to the ontological model; to define the starting requirements; and, fundamentally, its scope. In order to define the latter, Competence Questions[28] are identified, i.e., a list of questions that the Knowledge Base constructed from the ontological model must be able to respond. Following some 
of the questions identified in SportsWoman are presented:

- CQ1. Is the sportswoman user at risk of any disease?

- CQ2. Is there any recommendation for the sportswoman user?

- CQ3. At what stage of the menstrual cycle is the sportswoman user?

- CQ4. What is the hemoglobin level of the sportswoman user according to the last record?

- CQ5. What is the BMI of the sportswoman user?

Once the scope of the semantic model was determined, the Conceptualization Phase must be tackled. The objective of this phase is to organize and to structure the existing knowledge about the domain of interest. This phase is the most complex one of the entire ontology construction process. It involves the completion of several tasks, including the construction of a Glossary of Terms, the identification of Ontology Design Patterns, the identification of Concepts and Concept Hierarchy, and the identification of Binary Relations.

The outcome of this phase is a conceptual model easily recognizable by experts in the domain. Besides, it should facilitate the construction of a Knowledge Base with facts that allow answering to the Competence Questions. The conceptual model is represented utilizing the tabular descriptions of the concepts identified and graphs that can be easily interpreted by domain experts who may not be familiar with the notation and mathematical formalisms of knowledge representation. To facilitate the interpretation of the model by these experts, and according to the UPON methodology, UML diagrams were used to allow domain experts to review in a visual, simple and clear way the key relations established among concepts included within the proposed model.

Figure 5 shows a partial view of the obtained ontology. The reader can note that the central element of the model is the concept that represents the sportswoman. This is due to the fact that, after all, she is the central user of the system and the target for the recommendations or alerts generated through the Recommendation Manager. Following the design guidelines of the system, the woman user must be characterized anonymously; therefore, the ontology does not contain information on the name or any other universal identifier for the women. Instead, the identifier provided by the external OAuth server is used.

In order to characterize the sportwoman, three basic categories of information were modelled:

1) Profile. It supports data that characterizes the state and general context of the sportswoman through four factors:

a) Health: biometric information and daily routines (e.g., last weight and height recorded, hours of sleep or use of contraceptives).

b) Sport: information about practised sports and training habits (e.g., years of sports practice or training days per week).

c) Menstrual cycle: information about the history and average characterization of menstrual cycles (e.g., age of the first period, the regularity of the cycle or its usual duration). d) Symptomatology: information about the symptoms of the cycle perceived as usual (e.g., common pains, mood swings or fatigue).

2) HistoricalRecods. They allow having records on the history and evolution of the sportswoman over time. Two types of records are contemplated: a) Measures, physical evolution information (e.g., height, weight or temperature); and b) Analytics, information about blood tests (hemoglobine, hematocrit, ferritin, transferrin, etc.).

3) MenstrualCycle. It supports the identification of the main stages of each menstrual cycle. The phases that compose it (i.e., follicular, ovulation and lutea) and the associated particular symptomatology (e.g., pain, stress and bleeding).

Taking advantage of the modelling of these concepts and their relationships, the Recommendation Manager can infer new alerts and recommendations for the sportswoman user. The main result of the inference processes are instances of the HealthCondition and Recommendation classes, which have been included in the ontological model as represented in Figure 5:

- HealthCondition. This conceptual element represents the medical risk conditions for sportswomen. These conditions include an identifier ( $s w$ : mesh property) that links to the Medical Subject Headings (MeSH) RDF (e.g., the Anemia condition is linked to the URI http : //id.nlm.nih.gov/mesh/M0001119). This dataset is a linked data representation of the $\mathrm{MeSH}$ biomedical vocabulary produced by the US National Library of Medicine. With this URI, the system can consult reliable and detailed information about the referred condition. This linkage of the information managed by the SportWoman platform with external databases of recognized prestige makes the application of semantic enrichment techniques possible in further developments. The authors already applied this kind of techniques in several projects [29], [30] with satisfactory results that allowed to improve the recommendation processes.

- Recommendation. This element represents the suggestions generated by the domain experts. Recommendations can be classified according to their type or to the considered context (e.g., training or nutritional).

The conceptual model obtained is the starting artefact of the Coding Phase. Although, our research group typically addresses a Formalization Phase (in which the conceptual model is refined using mechanisms from Formal Logic) before the Coding Phase; in this platform, this was not the case as there were no complex ontological commitments identified in the semantic model. In the Coding Phase, the ontological model is coded using a knowledge representation language, particularly a Description Logic-based language (in our case RDF/OWL), so that an inference engine can handle it. Protégé [31] was selected as the most convenient ontology construction tool due to its versatility, simplicity and availability.

Once the model is built, it is required to evaluate it. This process is carried out in the Evaluation Phase. Although the real functional utility of the ontological model can only be 


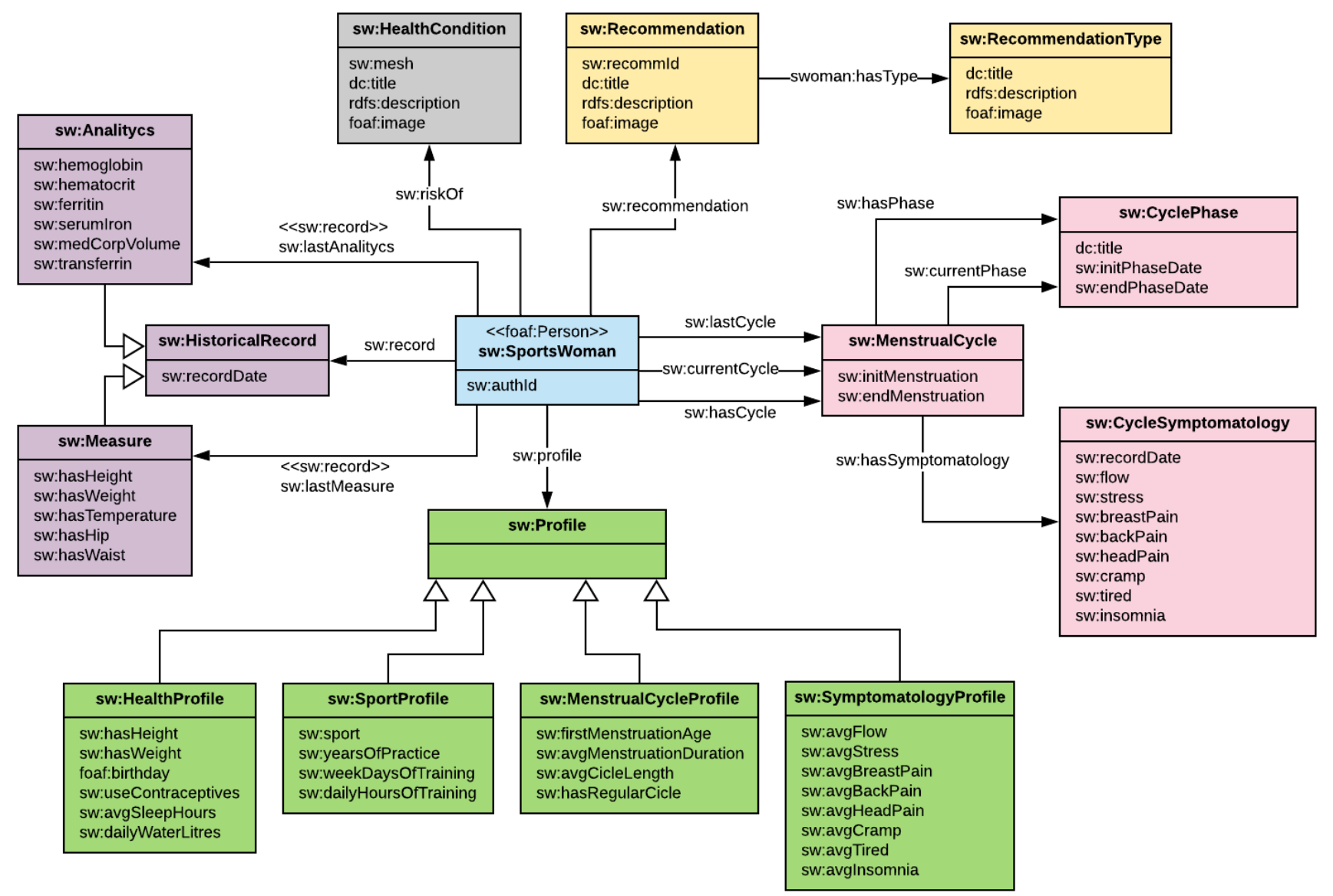

Fig. 5. Partial view of the SportsWoman ontology model.

assessed by tests with a final system that makes use of the ontology (in our case the SportsWoman platform), it is convenient, before reaching the production stage, to verify that the ontological concepts, their relationships and entailments identified allow to answer to the Competence Questions as defined in the Specification Phase.

This involves the transformation of each of these questions, expressed in natural language, into a semantic query language that can be interpreted by an inference engine. The Competence Questions, expressed in our case in SPARQL [32], must make use exclusively of the terms defined in the ontology. As an example, the following query, expressed in SPARQL, allows answering the competence question CQ1.

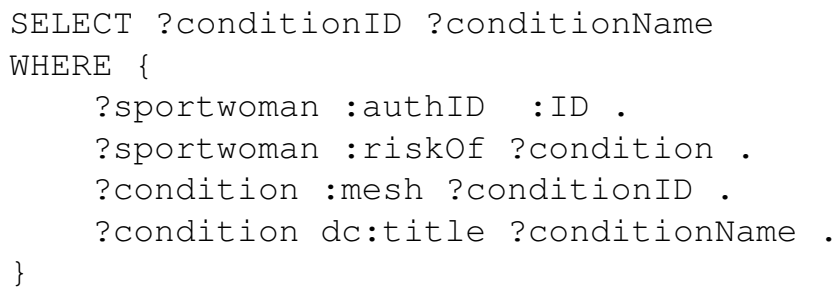

Correspondingly, the following SPARQL code allows dealing with Competence Question CQ3.

SELECT ?currentPhaseName

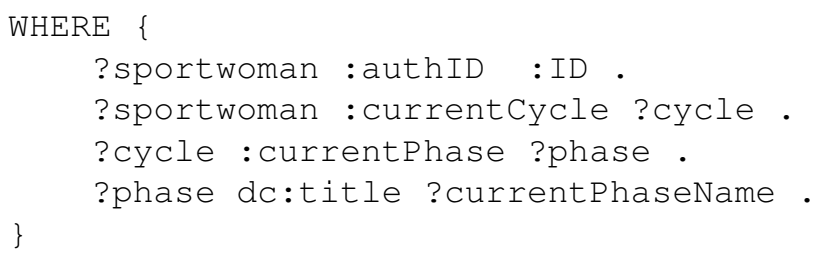

\section{VALIDATION}

To validate the usability of the proposed platform, a group of athletes affiliated with the University of Vigo FCCED centre were invited to participate in a pilot test of the platform. These women, who regularly practice sports, were able to use the tool developed for three weeks. After this period, the athletes completed the popular and well-known System Usability Scale (SUS) questionnaire [33]. SUS is a 10-item questionnaire that uses a 5-level Likert scale (from $1=$ "strongly disagree" to $5=$ "strongly agree"). The questionnaire combines positive items (in particular odd items) and negative items (even items). For positive items, the best possible response, from the point of view of usability, is reached when responding with a 5; and, conversely, for negative items, the best possible answer is 1.34 athletes answered the questionnaire. In Table III the different statistics obtained after the analysis of these answers are presented. At first glance, the results allow inferring that 
the perception of athletes on the usability of the platform is clearly high. As the reader can see in the table, the odd items concentrate answers between 5 and 4, while the even items concentrate the responses between 1 and 2. In particular, the resultant SUS score obtained was 86 , which corresponds to a range of acceptable usability. That value is associated with a grade $\mathrm{B}$, according to the scale proposed in [33].

To estimate the reliability of the SUS Score, the Cronbach's alpha was calculated from the answers obtained. Its value was 0.96 , surpassing the barrier of 0.9 , which corresponds to a excellent internal consistency of the answers [34].

\section{CONCLUSIONS}

In this article it has been presented SportsWoman, a telematics platform conceived as an Expert System that facilitates the collection of data related to the menstrual cycle and emulates the duty of a training assistant providing recommendations that take into account the menstrual cycle for the sports domain.

Today, this type of coaching service is only available to elite athletes, who can take advantage of personal trainers. SportsWoman aims to democratise this situation by making available to non-elite sports professionals and amateurs women the possibility of having personalised suggestions. At the same time, SportsWoman can be a useful resource for coaches to improve their work.

To carry out this task, SportsWoman codes the knowledge of specialists in the form of inference rules that generate these recommendations. Currently, SportsWoman's knowledge base contains approximately 50 rules of this nature. Despite not being a large number, the platform has shown to be solvent, as proven by the test group, a group of women regular practitioners of sports.

The platform has been designed so that the incorporation of new rules and new monitoring parameters is simple. This will facilitate the growth of the knowledge base as new recommendations and rules of interest to users are identified.

The authors are currently working on an extension of the platform that incorporates functionalities to adapt and customize the specific training plans of women athletes taking into account the recommendations and entities generated by the system. Likewise, the use of wrist-worn wearables [35] devices is being studied in order to automatically register the sport and fitness activities performed by the users.

In the future, it is expected to be able to use Machine Learning techniques in the platform. In particular, the possibility of including inductive inference techniques is planned to generate automatically new rules from the data collected. These new rules must be validated, in any case, by the specialists before forming part of the production rule set.

\section{ACKNOWLEDGMENT}

This work has been partially funded by the Spanish EAI and ISCIII and the ERDF "A way of making Europe" under projects TIN2016-80515-R (AEI/EFRD, EU) and PI16/00788 (CWB, MABM, LAS, JSV).

\section{REFERENCES}

[1] A. B. Loucks, "Effects of exercise training on the menstrual cycle: existence and mechanisms." Medicine and science in sports and exercise, vol. 22, no. 3, pp. 275-280, 1990.

[2] T. Dušek, "Influence of high intensity training on menstrual cycle disorders in athletes," Croat Med J, vol. 42, no. 1, pp. 79-82, 2001.

[3] N. F. Kishali, O. İmamoglu, D. Katkat, T. Atan, and P. Akyol, "Effects of menstrual cycle on sports performance," International Journal of Neuroscience, vol. 116, no. 12, pp. 1549-1563, 2006.

[4] N. Ozbar, F. C. Kayapinar, K. Karacabey, and R. Ozmerdivenli, "The effect of menstruation on sports women's performance," Studies on Ethno-Medicine, vol. 10, no. 2, pp. 216-220, 2016.

[5] J. M. Santos-Gago, L. M. Álvarez-Sabucedo, R. González-Maciel, V. M. Alonso-Rorís, J. L. García-Soidán, C. Wanden-Berghe, and J. Sanz-Valero, "Towards a personalised recommender platform for sportswomen," in World Conference on Information Systems and Technologies (WorldCist 2019). Springer, 2019, pp. 504-514.

[6] "Female fitness - women workout," Mobile App at https://play.google.com/store/apps/details?id=women.workout.female.fitness.

[7] "Workout for women: Fitness app," Mobile App at https://itunes.apple.com/us/app/workout-for-women-fitnessapp/id83928568.

[8] "Women workout: Home fitness, exercise \& burn fat," Mobile App at https://itunes.apple.com/us/app/women-workout-exerciseby/id909610529.

[9] "Fitbit homepage," Website available at https://www.fitbit.com/.

[10] D. Kosecki, "One of your most requested features is here! introducing female health tracking," Web blog available at https://blog.fitbit.com/female-health-tracking/.

[11] "Period tracker, ovulation calendar \& fertility," Mobile App at https://play.google.com/store/apps/details?id=periodtracker.pregnancy.ovulationtracker.

[12] "Period tracker flo, pregnancy \& ovulation calendar," Mobile App at https://play.google.com/store/apps/details?id=org.iggymedia.periodtracker

[13] "Period tracker: Monthly cycles," Mobile App at https://itunes.apple.com/us/app/period-tracker-monthlycycles/id368868193

[14] S. Sohda, K. Suzuki, and I. Igari, "Relationship between the menstrual cycle and timing of ovulation revealed by new protocols: analysis of data from a self-tracking health app," Journal of medical Internet research, vol. 19, no. 11, p. e391, 2017.

[15] A. Biørn-Hansen, T. A. Majchrzak, and T.-M. Grønli, "Progressive web apps for the unified development of mobile applications," in International Conference on Web Information Systems and Technologies. Springer, 2017, pp. 64-86.

[16] R. Akerkar and P. Sajja, Knowledge-based systems. Jones \& Bartlett Publishers, 2010.

[17] J. Liebowitz, The handbook of applied expert systems. cRc Press, 2019.

[18] A. Cañas-Rodriguez, J. M. Santos-Gago, L. E. Anido-Rifón, and R. Pérez-Rodrıguez, "A recommender system for non-traditional educational resources: a semantic approach," Journal of Universal Computer Science, vol. 21, no. 2, pp. 306-325, 2015.

[19] V. M. Alonso-Rorís, L. M. Álvarez-Sabucedo, J. M. Santos-Gago, and M. Ramos-Merino, "Towards a cost-effective and reusable traceability system. a semantic approach," Computers in Industry, vol. 83, pp. 1-11, 2016.

[20] M. Cervera-Peris, V. M. Alonso-Rorís, J. M. Santos-Gago, L. M. Álvarez-Sabucedo, C. Wanden-Berghe, and J. Sanz-Valero, "Management of the general process of parenteral nutrition using mhealth technologies: Evaluation and validation study," JMIR mHealth and uHealth, vol. 6, no. 4, p. e79, 2018.

[21] D. Hardt, RFC 6749. The OAuth 2.0 Authorization Framework, IETF Std., 2012. [Online]. Available: https://tools.ietf.org/html/rfc6749

[22] J. M. Santos-Gago, L. E. Anido-Rifón, and M. Llamas-Mistal, "Design of a semantic web-based brokerage architecture for the e-learning domain. a proposal for a suitable ontology," in 35th IEEE Frontiers in Education Conference. IEEE, 2005, pp. S3H-S3H.

[23] R. Miguez-Pérez, J. M. Santos-Gago, and L. E. Anido-Rifón, "A holistic framework to support ict-based early childhood education processes," in 39th IEEE Frontiers in Education Conference. IEEE, 2009, pp. 1-6.

[24] M. Fernández-López, A. Gómez-Pérez, and N. Juristo, "Methontology: from ontological art towards ontological engineering," in Spring Symposium on Ontological Engineering of AAAI. Stanford University, California, 1997, pp. 33-40. 
TABLE III

RESPONSES TO THE SUS QUESTIONNAIRE

\begin{tabular}{|c|c|c|c|c|}
\hline & Item & Mean & Mode & SD \\
\hline Q1 & I think that I would like to use this app frequently & 4.588 & 5 & 0.492 \\
\hline Q2 & I found the app unnecessarily complex & 2.029 & 2 & 0.664 \\
\hline Q3 & I thought the app was easy to use & 4.618 & 5 & 0.486 \\
\hline Q4 & $\begin{array}{l}\text { I think that I would need the support of a technical person to be able to use } \\
\text { this app }\end{array}$ & 1.206 & 1 & 0.404 \\
\hline Q5 & I found the various functions in this app were well integrated & 4.353 & 4 & 0.476 \\
\hline Q6 & I thought there was too much inconsistency in this app & 2.235 & 2 & 0.644 \\
\hline Q7 & I would imagine that most people would learn to use this app very quickly & 4.676 & 5 & 0.468 \\
\hline Q8 & I found the app very cumbersome to use & 1.971 & 2 & 0.514 \\
\hline Q9 & I felt very confident using the app & 4.765 & 5 & 0.424 \\
\hline Q10 & I needed to learn a lot of things before I could get going with this app & 1.118 & 1 & 0.322 \\
\hline
\end{tabular}

[25] M. C. Suárez-Figueroa, A. Gómez-Pérez, and M. Fernández-López, "The neon methodology for ontology engineering," in Ontology Engineering in a Networked World. Springer Berlin Heidelberg, 2012, pp. 9-34.

[26] H. S. Pinto, S. Staab, and C. Tempich, "Diligent: Towards a finegrained methodology for distributed, loosely-controlled and evolving," in Proceedings of the 16th European Conference on Artificial Intelligence (ECAI 2004), vol. 110, 2004, p. 393.

[27] A. De Nicola, M. Missikoff, and R. Navigli, "A proposal for a unified process for ontology building: Upon," in International Conference on Database and Expert Systems Applications. Springer, 2005, pp. 655664.

[28] M. Grüninger and M. S. Fox, "Methodology for the design and evaluation of ontologies," 1995.

[29] V. M. Alonso-Rorís, J. M. Santos-Gago, R. Pérez-Rodríguez, C. RivasCosta, M. Gómez-Carballa, and L. E. Anido-Rifón, "Information extraction in semantic, highly-structured, and semi-structured web sources," Polibits, no. 49, pp. 69-76, 2014.

[30] V. M. Alonso-Rorís, R. Míguez-Pérez, J. M. Santos-Gago, and L. M. Álvarez-Sabucedo, "A semantic enrichment experience in the early childhood context," in 2012 Frontiers in Education Conference Proceedings. IEEE, 2012, pp. 1-6.

[31] "Protégé: A free, open-source ontology editor and framework for building intelligent systems," Website available at https://protege.stanford.edu/.

[32] S. Harris and A. Seaborne, SPARQL 1.1 Query Language, W3C Std., Mar. 2013. [Online]. Available: https://www.w3.org/TR/2013/RECsparql11-query-20130321/

[33] A. Bangor, P. Kortum, and J. Miller, "Determining what individual sus scores mean: Adding an adjective rating scale," Journal of usability studies, vol. 4, no. 3, pp. 114-123, 2009.

[34] J. A. Gliem and R. R. Gliem, "Calculating, interpreting, and reporting cronbach's alpha reliability coefficient for likert-type scales." Midwest Research-to-Practice Conference in Adult, Continuing, and Community Education, 2003.

[35] J. M. Santos-Gago, M. Ramos-Merino, S. Vallarades-Rodriguez, L. M. Álvarez-Sabucedo, M. J. Fernández-Iglesias, and J. L. García-Soidán, "Innovative use of wrist-worn wearable devices in the sports domain: A systematic review," Electronics, vol. 8, no. 11, p. 1257, 2019. 